\title{
Use of a 2-Dimensional Ionization Chamber Array to Measure Head Leakage of a Varian Truebeam ${ }^{\circledR}$ Linear Accelerator
}

\author{
Sameer Taneja*, Jose R. Teruel, Lei Hu, Jinyu Xue, David Barbee \\ Department of Radiation Oncology, NYU Langone Health, New York, USA \\ Email: ^sameer.taneja@nyulangone.org
}

How to cite this paper: Taneja, S., Teruel, J.R., Hu, L., Xue, J. and Barbee, D. (2020) Use of a 2-Dimensional Ionization Chamber Array to Measure Head Leakage of a Varian Truebeam ${ }^{\otimes}$ Linear Accelerator. International Journal of Medical Physics, Clinical Engineering and Radiation Oncology, 9, 87-95.

https://doi.org/10.4236/ijmpcero.2020.93009

Received: May 19, 2020

Accepted: June 12, 2020

Published: June 15, 2020

Copyright $\odot 2020$ by author(s) and Scientific Research Publishing Inc. This work is licensed under the Creative Commons Attribution International License (CC BY 4.0).

http://creativecommons.org/licenses/by/4.0/ (c) (i) Open Access

\begin{abstract}
The purpose of this work is to evaluate the use of a two-dimensional (2D) planar ion chamber array to characterize leakage radiation from the head of the linear accelerator. Ion chamber arrays provide a benefit over a singular ion chamber measurement as they allow for the measurement of a larger area in order to isolate the point of maximum leakage dose and the small size of each individual ion chamber minimizes volume-averaging effects. A Varian Truebeam ${ }^{\circledR}$ undergoing acceptance testing was used for all measurements. The gantry was wrapped in Portal Pack for Localization (PPL) radiographic film in order to isolate the location of maximum leakage. A calibration curve was developed and used to determine dose-to-film. An Ion Chamber Profiler (IC Profiler ${ }^{\mathrm{m}}$ ) manufactured by Sun Nuclear Corporation was used to confirm measurements by the PPL film. All measurements were normalized to leakage at $100 \mathrm{~cm}$ from the target relative to the central axis. Three points were investigated with the IC Profiler, including the top of the gantry, the Varian logo, and the side of the gantry. For the three locations, respectively, the PPL film and the IC profiler were measured $0.142 \%$ and $0.131 \%, 0.036 \%$ and $0.030 \%$, and $0.014 \%$ and $0.019 \%$. The good agreement between the PPL film and the IC Profiler provides confidence in the use of a more efficient and accurate ion chamber array for head leakage measurements.
\end{abstract}

\section{Keywords}

Gantry Leakage, Linear Accelerator Commissioning, Ionization Chamber Array

\section{Introduction}

During the acceptance of a new linear accelerator, the adequacy of the shielding 
of the treatment head is evaluated through the measurement of leakage radiation. According to the National Council on Radiation Protection (NCRP) report 49 , the recommended magnitude of leakage radiation, defined at 1 meter from the source, should not exceed $0.1 \%$ of the exposure along the primary axis at the 1 meter from the source for a megavoltage linear accelerator [1]. A more recent report from the NCRP defines leakage radiation as radiation measured outside of the maximum field size produced. The leakage radiation limit is $0.2 \%$ of the absorbed dose rate on the central axis and at the same treatment depth [2].

Leakage radiation from the accelerator head is of clinical importance as peripheral dose for structures with low dose tolerances may affect patient treatments. Peripheral dose consists of three sources of radiation, including patient scatter, collimator scatter, and head leakage [3]. At greater distances from the treatment field, around $20 \mathrm{~cm}$ (the distance depends on treatment field parameters, beam modulation, and beam energy), head leakage dominates peripheral dose [4]. Leakage measurements performed during accelerator acceptance can be used to characterize collimator and head scatter doses, and confirm that the head leakage falls within NCRP recommendations [5].

In addition, measurements of head leakage are important to assess the shielding effectiveness of a linear accelerator vault. Radiation outside of the primary beam is dominated by head leakage, and the thickness of the secondary barrier is directly affected by the magnitude of this radiation [6].

According to a recent survey, only about $54 \%$ of the sixty-five physicists that participated perform a leakage survey during acceptance testing (https://www.surveymonkey.com/results/SM-YRG2SBGJ/). The low number of leakage measurements is mainly due to no outlined method and the cost and availability of detectors. As a result, the manufacturer's specifications of leakage are often used for shielding calculations and the improvement of the head construction and the shipment of the head as one unit (instead of building the head in the vault) for modern linear accelerators improves the consistency and accuracy of the manufacturer's specifications.

Previous studies have looked to characterize head leakage from various linear accelerators. Most studies determined leakage dose at cardinal angles using Monte Carlo [7] and measurements using point detectors such as radiographic film, thermoluminescent detectors, diodes, and ionization chambers [3] [4] [8] [9] [10]. The conventional method for determining head leakage is to wrap the gantry in film to isolate the point of maximum leakage, and use and ion chamber to verify the dose [11].

The challenges of using an ion chamber to measure leakage dose include 1) a standard farmer chamber could be larger than the leakage radiation identified by the film, resulting in partial volume effects, and 2) positioning the farmer chamber in the maximum leakage spot requires an iterative approach to ensure that the chamber is capturing maximum leakage dose. An ion chamber array has the advantage over a point detector because it can measure dose in a plane and each 
chamber has a small active volume. The purpose of this work is to investigate the accuracy of using a commercial ion chamber array to measure leakage dose and compare the results with film measurements. Through this comparison of measurement accuracy presented in the upcoming sections, a new and more accessible method for head leakage determination is discussed.

\section{Methods}

A Varian Truebeam ${ }^{\circledR}$ (Varian Medical Systems, Palo Alto, CA) was recently installed at the New York University (NYU) Perlmutter Cancer Center at Sunset Park (Brooklyn, NY) and was used for all measurements. All leakage measurements were taken during the acceptance and commissioning of the linear accelerator using a step method, which included determining the point along the linear accelerator head of maximum leakage using film and confirming the measured dose with an ionization chamber array.

Oncology portal pack for localization (PPL) film manufactured by Carestream Health (Rochester, NY) was used to determine the location on the gantry head where the maximum leakage was present. According to the manufacturer, PPL film has a responsive range of (0.25 to 5) cGy and an approximate saturation exposure $>10 \mathrm{cGy}$. A calibration curve was produced for the PPL film by delivering $(1,1.5,2,2.5,3,4,6,8,10)$ cGy to film (Figure 1). Post irradiation, the films were exposed at the NYU department of radiation safety, and read out using a clamshell densitometer manufactured by Fluke Biomedical (Solon, $\mathrm{OH}$ ).

Calculations of the leakage of the linear accelerator head were performed using Equation (1),

$$
\text { Leakage }(\%)=\frac{D_{\text {Leakage }}}{D_{\text {Norm }}} \times 100 \%
$$

where $D_{\text {Leakage }}$ and $D_{\text {Norm }}$ are the measured leakage dose and central axis dose

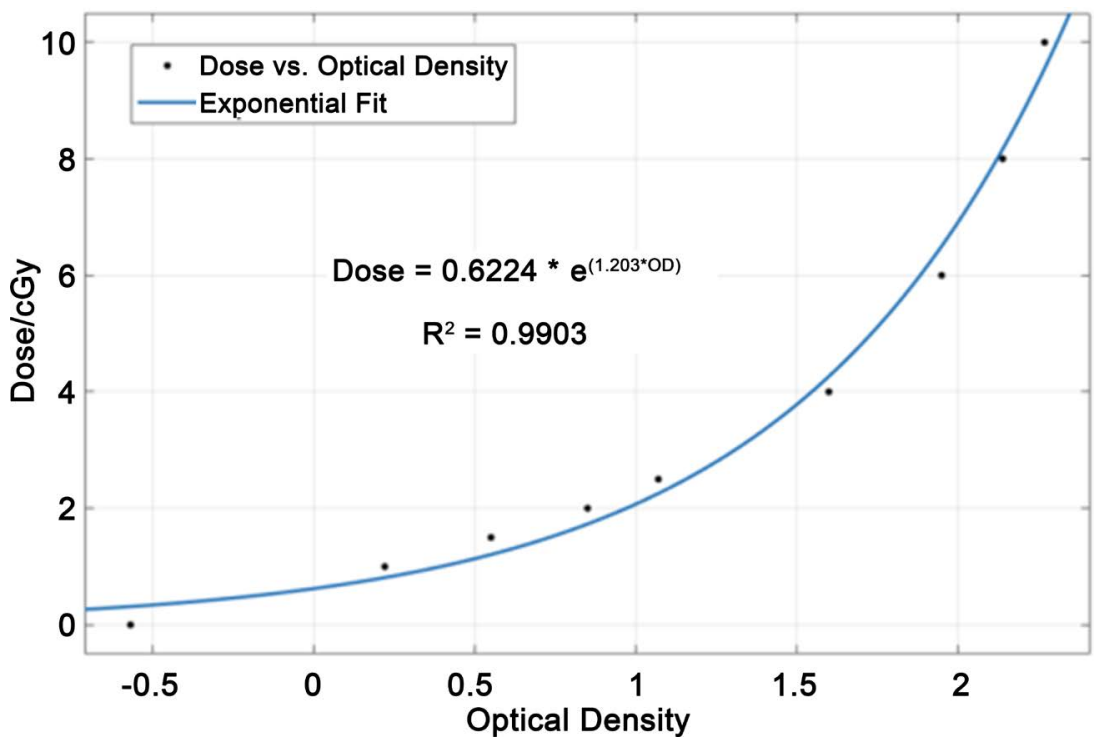

Figure 1. Calibration curve for the Carestream PPL radiographic film. 
used for output normalization, respectively. The following sections described the methods for measuring leakage and central axis output.

PPL films were wrapped around the linear accelerator gantry head (Figure 2) and $300 \mathrm{MU}$ were delivered, which was calculated to be the optimal dose based on the manufacturer's leakage measurements, the distance from the target to the PPL film, and the responsive range of the film. Dose was delivered using an energy of $15 \mathrm{MV}$, a gantry angle of $90^{\circ}$, jaws set to a minimum field size of $(0.5 \times$ $0.5) \mathrm{cm}^{2}$, and with the multi leaf collimators (MLCs) fully extended. The location and orientation on the gantry head for each PPL film was recorded during the irradiation. The maximum optical density was measured on each film using the densitometer and the maximum dose was determined using the calibration curve, which was used to calculate the dose at 1 meter away from the gantry head. The dose was normalized to $300 \mathrm{cGy}$, which was the maximum dose delivered along the central axis in $300 \mathrm{MU}$ at reference conditions of 1 meter from the gantry head using a $(10 \times 10) \mathrm{cm}^{2}$ field size and with the MLCs retracted. Leakage measurements were performed using an Ion Chamber Profiler [12] (IC Profiler $^{\mathrm{Tw}}$ ) manufactured by Sun Nuclear Corporation (Melbourne, Fl) to confirm the points of maximum leakage radiation determined by the PPL film. The IC Profiler consists of an array of 251 ion chambers arranged with an active volume for each detector of $0.046 \mathrm{~cm}^{3}$ and a spacing of $0.5 \mathrm{~cm}$ between detectors on the principal axes ( $\mathrm{x}$ and $\mathrm{y}$ ) and $0.7 \mathrm{~cm}$ on the diagonal axes. All detectors are located at a water equivalent depth of $0.9 \mathrm{~cm}$ and the maximum field size covered is $(32 \times 32) \mathrm{cm}^{2}$. The IC Profiler was placed along the axis of maximum leakage, which was determined by the film. The IC Profiler was placed at a measured distance of $100 \mathrm{~cm}$ from the accelerator's target, which was located using the manufacturer's schematics (Figure 3). A measurement of maximum
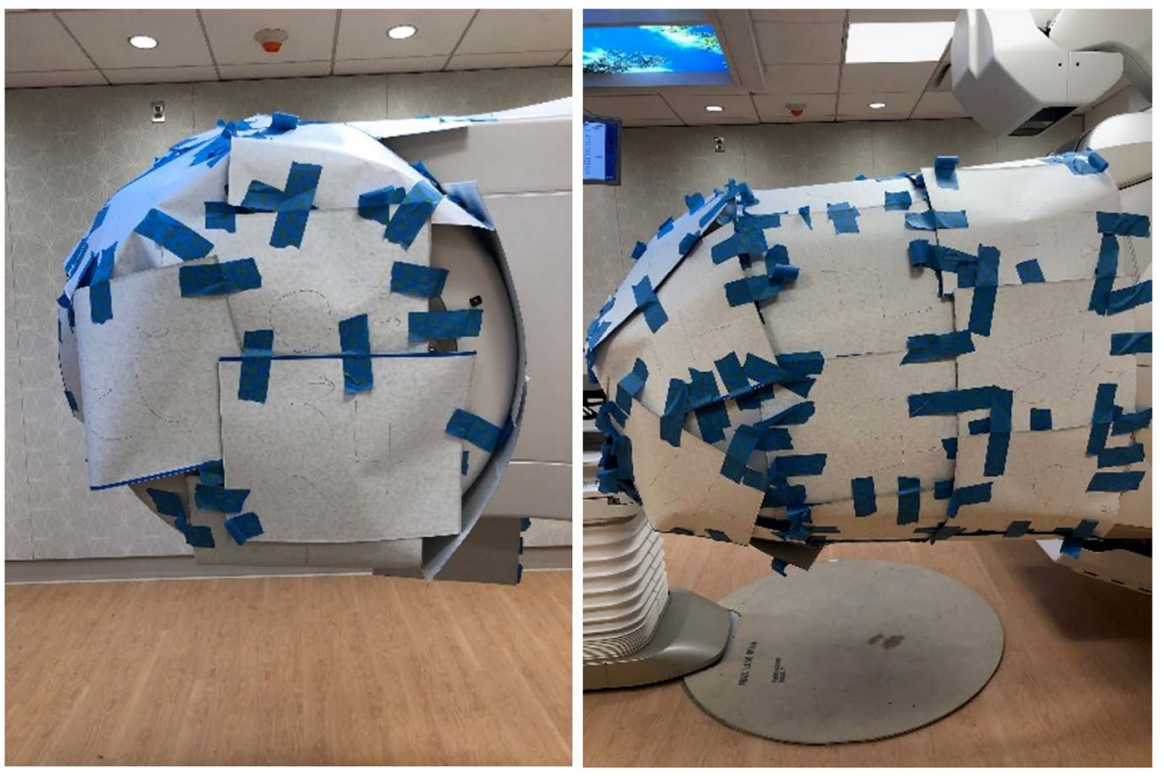

Figure 2. Photographs of the Truebeam ${ }^{\circledR}$ linear accelerator gantry head wrapped with PPL film (left: collimator side, right: source side). 
dose was completed over the course of three separate measurement sessions, allowing for the characterization of setup uncertainty and reproducibility. Measurements were normalized to a measurement of the IC Profiler placed at 100 $\mathrm{cm}$ from the source along the central axis $(10 \times 10) \mathrm{cm}^{2}$ field size, $100 \mathrm{MU}$ delivered, and MLCs retracted.

\section{Results}

A total of 35 films were placed along the gantry head, and their locations were determined using coordinate system with angular and azimuthal angles (Figure 4).
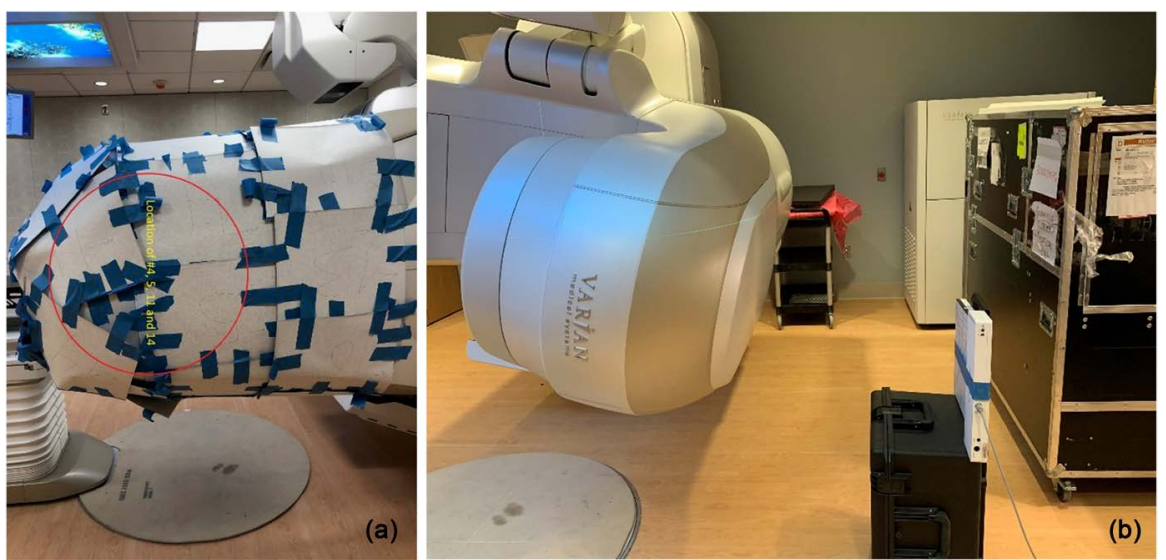

Figure 3. (a) Location of the maximum leakage from the head as determined by PPL films, and (b) measurement setup of leakage at $100 \mathrm{~cm}$ from the target using the IC Profiler.

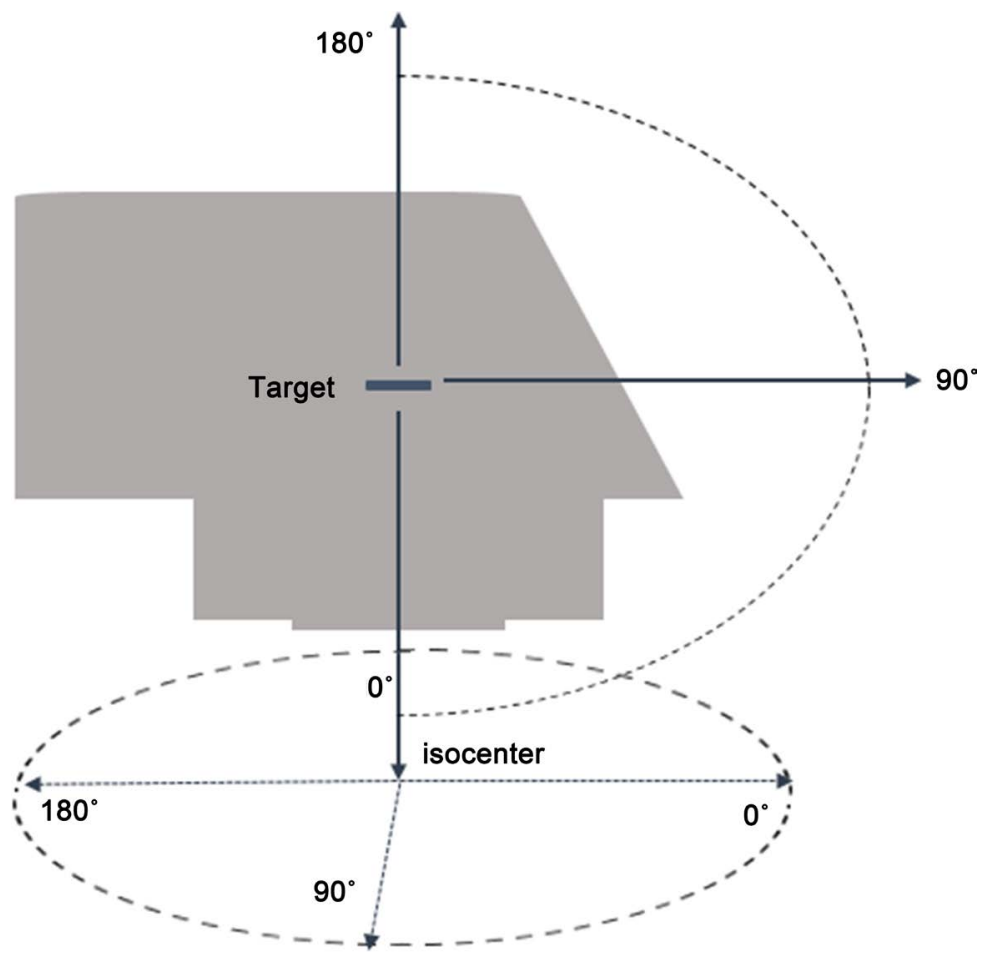

Figure 4. Angular representation of the linear accelerator gantry head used for film localization. 
A subset of the PPL films that showed a visible exposure and their associated leakage dose location on the gantry head are shown in Table 1 . The dose at 100 $\mathrm{cm}$ from the target was calculated using the $\mathrm{r}$-squared falloff and the leakage at $100 \mathrm{~cm}$ was determined by normalizing to $300 \mathrm{cGy}$, based on the $300 \mathrm{MU}$ delivered for the measurement and the output calibrated to $1 \mathrm{cGy} / \mathrm{MU}$. The maximum leakage dose was measured on film 4 , which was located at angular and azimuthal angles of $180^{\circ} / 0^{\circ}$ from the central axis. Maximum leakage measured on film ranged from $0.019 \%$ to $0.134 \%$ relative to the central axis, with the majority of films showing no exposure above background.

The IC Profiler was used to measure leakage dose to three locations determined by the PPL film, at $180^{\circ} / 0^{\circ}$ (Film no. 4 ), $90^{\circ} / 0^{\circ}$ (Film no. 7), and $0^{\circ} / 90^{\circ}$ (Film no. 3) from the central axis and using three independent setups. Figure 5 shows a representative result from the IC Profiler measurement placed at $100 \mathrm{~cm}$ from the target and at $180^{\circ} / 0^{\circ}$ from the central axis. Ion chamber measurements along the $\mathrm{x}$ - and $\mathrm{y}$-axis as well as the negative and positive diagonals are presented. The maximum dose was present on the positive diagonal and was measured as $1.33 \mathrm{cGy}$. This was normalized to the dose along the central axis to determine leakage.

Table 2 shows the comparison of leakage measurements from the IC Profiler and the PPL film at three locations. Three measurements with the IC Profiler were made at each location, and the Type A uncertainty was determined for the measurement. At $180^{\circ} / 0^{\circ}$, leakage was $0.142 \%$ and $0.130 \%$ for measurements by the IC Profiler and the PPL film, respectively. At $90^{\circ} / 0^{\circ}$, leakage was $0.036 \%$ and $0.030 \%$ measured by the IC Profiler and the PPL film, respectively. Finally, at $0^{\circ} / 90^{\circ}$, leakage was $0.014 \%$ and $0.019 \%$ measured by the IC Profiler and the PPL film, respectively. The differences between IC Profiler and film measurements were $0.008 \%, 0.006 \%$, and $-0.005 \%$ for the 3 locations.

Table 1. Measured optical density and corresponding dose for all PPL films with visible dose. Dose at $100 \mathrm{~cm}$ and leakage at $100 \mathrm{~cm}$ were calculated.

\begin{tabular}{cccccc}
\hline Film No. & $\begin{array}{c}\text { Location } \\
\text { (Angular/Azimuthal) }\end{array}$ & $\begin{array}{c}\text { Optical } \\
\text { Density }\end{array}$ & $\begin{array}{c}\text { Film dose } \\
(\mathrm{cGy})\end{array}$ & $\begin{array}{c}\text { Dose at } 100 \mathrm{~cm} \\
(\mathrm{cGy})\end{array}$ & $\begin{array}{c}\text { Leakage at } 100 \\
\mathrm{~cm}(\%)\end{array}$ \\
\hline 3 & $0^{\circ} / 90^{\circ}$ & 0.00 & 0.62 & 0.06 & 0.02 \\
$4^{*}$ & $180^{\circ} / 0^{\circ}$ & 1.64 & 4.48 & 0.40 & 0.13 \\
6 & $180^{\circ} / 315^{\circ}$ & -0.21 & 0.48 & 0.04 & 0.01 \\
$7^{*}$ & $90^{\circ} / 0^{\circ}$ & 0.20 & 0.79 & 0.07 & 0.02 \\
8 & $135^{\circ} / 90^{\circ}$ & 0.18 & 0.77 & 0.07 & 0.02 \\
11 & $180^{\circ} / 45^{\circ}$ & 1.10 & 2.34 & 0.21 & 0.07 \\
14 & $180^{\circ} / 0^{\circ}$ & 0.53 & 1.18 & 0.11 & 0.04 \\
18 & $180^{\circ} / 0^{\circ}$ & 0.22 & 0.81 & 0.07 & 0.02 \\
31 & $210^{\circ} / 0^{\circ}$ & -0.20 & 0.49 & 0.04 & 0.01 \\
\hline
\end{tabular}

${ }^{\star}$ Films used for validation of the IC Profiler. 


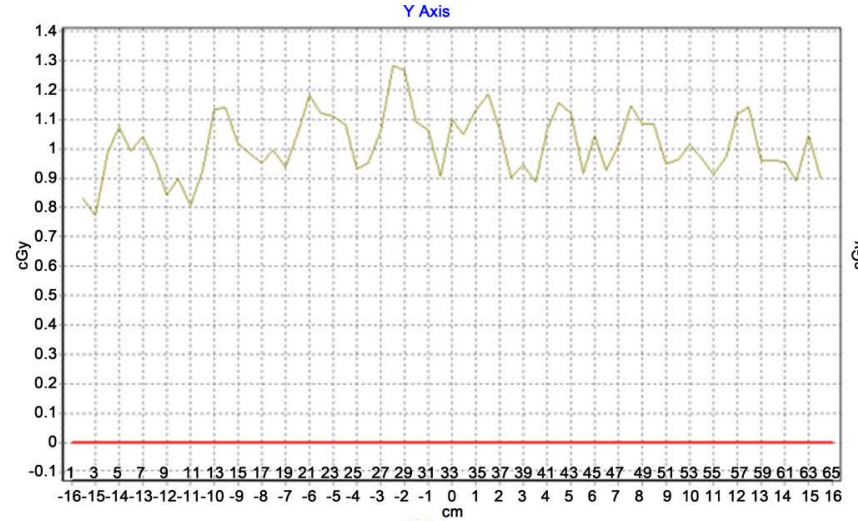

$\mathrm{x}$ Axis

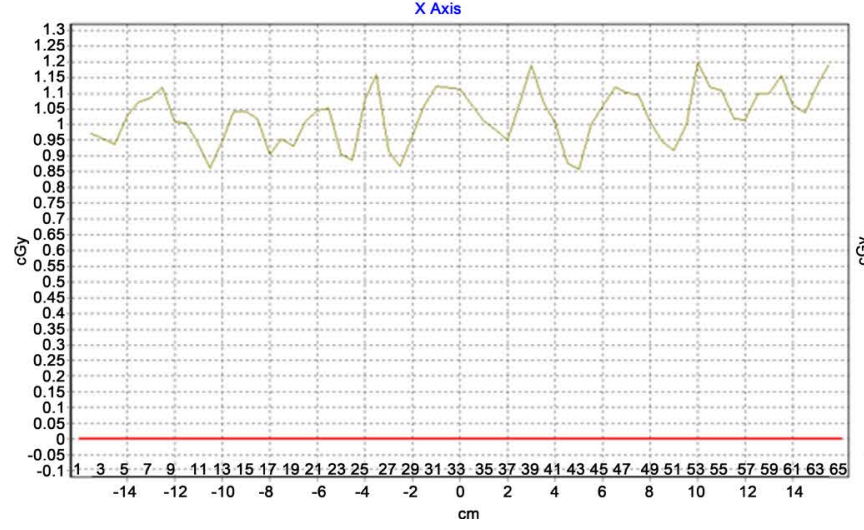

Figure 5. Results from the IC Profiler placed at $180^{\circ}$ from the central axis. Arrays along the $\mathrm{Y}$-axis, $\mathrm{X}$-axis, positive and negative diagonals are presented. The maximum dose delivered to the Profiler was 1.38 cGy measured along the positive diagonal.

Table 2. Results from the IC Profiler measurements and a comparison of measured leakage using the IC Profiler and the PPL film.

\begin{tabular}{ccccccc}
\hline Location & $\begin{array}{c}\text { Angular/ } \\
\text { Azimuthal }\end{array}$ & MUs & $\begin{array}{c}\text { Dose to IC } \\
\text { Profiler (cGy) }\end{array}$ & $\begin{array}{c}\text { \% Leakage } \\
\text { (IC Profiler) }\end{array}$ & $\begin{array}{c}\text { Uncertainty } \\
\text { (\%) }\end{array}$ & $\begin{array}{c}\text { \% Leakage } \\
\text { (PPL film) }\end{array}$ \\
\hline Central axis (Open)* & & 100 & 90.8 & - & & - \\
Top of gantry & $180^{\circ} / 0^{\circ}$ & 1000 & 1.29 & 0.142 & 3.75 & 0.1334 \\
Varian Logo & $90^{\circ} / 0^{\circ}$ & 1000 & 0.328 & 0.036 & 9.12 & 0.020 \\
Side of Gantry & $0^{\circ} / 90^{\circ}$ & 1000 & 0.123 & 0.014 & 2.15 & 0.019 \\
\hline
\end{tabular}

*Used for IC Profiler normalization.

\section{Discussion}

Characterization of leakage radiation from a linear accelerator is important because of its impacts on peripheral dose to the patient, shielding calculations, and evaluation of the construction of the head of a linear accelerator. Current methods of leakage determination using film can be time consuming, labor intensive, and costly. As a result, this work looked to verify the accuracy of a 2-D ion chamber array to measure leakage radiation by comparing leakage radiation measurements from the array to measurements completed using film.

Previous work has used point detectors to measure leakage radiation, typically at the cardinal angles. These measurements are limited based on the construc- 
tion of the detector and the placement of the detector relative to the gantry head. The IC Profiler provides a 2-D array of detectors that cover an area of $(32 \times 32)$ $\mathrm{cm}^{2}$. Ion chambers are placed along the principal and diagonal axes of the IC Profiler, which inherently provides limitations in measurements outside of those axes, but the IC profiler allows for improved measurement coverage compared with point measurements from ion chambers.

There has been limited work published on the energy-dependent response of the PPL film in megavoltage beams. Comparable radiographic films were investigated in work performed by Muench et al. [13], and it was found that variable response occurs at energies $<100 \mathrm{KeV}$ [14]. The relative contribution of low energy photons to leakage measurements is anticipated to be low, as leakage radiation is dominated by thick-target bremsstrahlung at large angles, and the leakage energy spectrum is similar to a useful megavoltage beam [15]. $15 \mathrm{MV}$ photons were used in this work and it is expected that the majority of the leakage measurement originated from photon penetration from the linear accelerator head where less shielding was present. In addition, this work calculated leakage at a distance of $100 \mathrm{~cm}$ from the target of a linear accelerator using PPL film by using an $r$-squared falloff factor. Based on the nature of leakage radiation, this was assumed the most accurate representation of dose falloff. This work validated the use of a commercial detector array for the measurement of head leakage by comparing the percent leakage to PPL film. Results showed good agreement between the IC Profiler and the PPL film, with a maximum difference of $0.008 \%$.

There are two main considerations for medical physicists when characterizing leakage radiation from the gantry head, 1) isolating the point of maximum leakage, and 2) accurately measuring the leakage radiation to determine if the accelerator meets NCRP recommendations. Ideally, a physicist will wrap the gantry head in film to visually determine the location of maximum leakage and confirm the dose using ion chamber measurements. The use of an ion chamber array presented in this work will not be able to isolate the point of maximum leakage, but will provide a more efficient method for determining the dose at $100 \mathrm{~cm}$ when compared with an ionization chamber.

Based on the results of the presented survey, only $54 \%$ of physicists perform leakage measurements during acceptance testing. Instead of not performing leakage measurements, it would be recommended to use an ion chamber array to determine dose at $100 \mathrm{~cm}$ from the target at a set of locations where there is less shielding on the gantry head. These measurements points can be determined using schematics from the manufacturer, and in some cases, previous measurements performed by the manufacturer. This method acknowledges the robustness of modern linear accelerator construction and installation, and that there are few locations on the gantry head construction that could produce leakage radiation when a linear accelerator is installed.

\section{Conflicts of Interest}

The authors declare no conflicts of interest regarding the publication of this paper. 


\section{References}

[1] National Council on Radiation Protection and Measurements (1976) NCRP Report No 49: Structural Shielding Design and Evaluation for Megavoltage X- and Gamma-Ray Radiotherapy Facilities: Recommendations of the National Council on Radiation Protection and Measurements.

[2] National Council on Radiation Protection and Measurements (1989) NCRP Report No 102: Medical X-Ray, Electron Beam and Gamma-Ray Protection for Energies up to $50 \mathrm{MeV}$ (Equipment Design, Performance and Use).

[3] Stern, R.L. (1999) Peripheral Dose from a Linear Accelerator Equipped with Multileaf Collimation. Medical Physics, 26, 559-563. https://doi.org/10.1118/1.598557

[4] Kase, K.R., Svensson, G.K., Wolbarst, A.B. and Marks, M.A. (1983) Measurements of Dose from Secondary Radiation outside a Treatment Field. International Journal of Radiation Oncology, Biology, Physics, 9, 1177-1183. https://doi.org/10.1016/0360-3016(83)90177-3

[5] Kry, S.F., et al. (2017) AAPM TG 158: Measurement and Calculation of Doses Outside the Treated Volume from External-Beam Radiation Therapy. Medical Physics, 44, e391-e429. https://doi.org/10.1002/mp.12462

[6] Jaradat, A.K. and Biggs, P.J. (2007) Measurement of the Leakage Radiation from Linear Accelerators in the Backward Direction for 4, 6, 10, 15, and $18 \mathrm{MV}$ X-Ray Energies. Health Physics, 92, 387-395. https://doi.org/10.1097/01.HP.0000252308.89839.7d

[7] Nelson, W.R. and LaRiviere, P.D. (1984) Primary and Leakage Radiation Calculations at 6, 10 and $25 \mathrm{MeV}$. Health Physics, 47, 811-818.

https://doi.org/10.1097/00004032-198412000-00001

[8] Jaradat, A.K. and Biggs, P.J. (2008) Measurement of the Neutron Leakage from a Dedicated Intraoperative Radiation Therapy Electron Linear Accelerator and a Conventional Linear Accelerator for 9, 12, 15(16), and 18(20) MeV Electron Energies. Medical Physics, 35, 1711-1717. https://doi.org/10.1118/1.2898144

[9] Van der Giessen, P.H. (1994) Calculation and Measurement of the Dose at Points outside the Primary Beam for Photon Energies of 6, 10, and 23 MV. International Journal of Radiation Oncology, Biology, Physics, 30, 1239-1246. https://doi.org/10.1016/0360-3016(94)90335-2

[10] Fair, H.I. (1992) A Method of Calculating Peripheral Dose Distributions of Photon Beams below 10 MV. Medical Physics, 19, 283-293.

https://doi.org/10.1118/1.596858

[11] IEC (2009) IEC 60601-2-1: Particular Requirements for the Basic Safety and Essential Performance of Electron Accelerators in the Range $1 \mathrm{MeV}$ to $50 \mathrm{MeV}$.

[12] Simon, T.A., Kozelka, J., Simon, W.E., Kahler, D., Li, J. and Liu, C. (2010) Characterization of a Multi-Axis Ion Chamber Array. Medical Physics, 37, 6101-6111. https://doi.org/10.1118/1.3505452

[13] Muench, P.J., Meigooni, S., Nath, R. and McLaughlin, W.L. (1990) Photon Energy Dependence of the Sensitivity of Radiochromic Film and Comparison with Silver Halide Film and LiF TLDs Used for Brachytherapy Dosimetry. Medical Physics, 18, 769-775. https://doi.org/10.1118/1.596630

[14] Pai, S., et al. (2007) TG-69: Radiographic Film for Megavoltage Beam Dosimetry. Medical Physics, 34, 2228-2258. https://doi.org/10.1118/1.2736779

[15] Karzmark, C.J. (1980) Radiological Safety Aspects of the Operation of Electron Linear Accelerators, by W. P. Swanson. Medical Physics, 7, 393-394.

https://doi.org/10.1118/1.594816 